\title{
Traduire
}

Une eutre perspective sur $r$ tatadciction

Revue française de la traduction

$220 \mid 2009$

Organisations internationales | Bicentenaire de Louis Braille

\section{Introduction à la Traductologie, Mathieu Guidère}

Lynne Franjié

\section{OpenEdition}

Journals

Édition électronique

URL : http://journals.openedition.org/traduire/394

DOI : $10.4000 /$ traduire.394

ISSN : 2272-9992

Éditeur

Société française des traducteurs

Édition imprimée

Date de publication : 15 juin 2009

Pagination : 71-72

ISSN : 0395-773X

\section{Référence électronique}

Lynne Franjié, «Introduction à la Traductologie, Mathieu Guidère », Traduire [En ligne], 220 | 2009, mis en ligne le 12 novembre 2013, consulté le 22 septembre 2020. URL : http://journals.openedition.org/ traduire/394 ; DOI : https://doi.org/10.4000/traduire.394 


\section{Compte-rendu d'ouvrage \\ Introduction à la Traductologie, \\ Mathieu Guidère}

\section{Lynne Franjié}

Université Stendhal-Grenoble 3

Dans son ouvrage Introduction à la traductologie, paru récemment dans la collection Traducto des éditions De Boeck, Mathieu Guidère, professeur de traductologie à l'École d'Interprètes de Traducteurs (ETI) de l'Université de Genève, dresse un panorama d'un domaine d'études ancien et nouveau à la fois, la traductologie. II s'agit d'une synthèse exhaustive, structurée et étayée des recherches menées sur la traduction notamment dans le monde francophone. Les théories et les approches élaborées sur la traduction y sont toutes réunies en mettant en évidence leurs convergences et leurs divergences et la manière dont elles se complètent ou s'affrontent.

L'ouvrage propose une vision novatrice et ambitieuse de la traduction et de la traductologie en montrant l'évolution qu'a connue la traduction en passant d'un simple transfert linguistique à une opération de communication multilingue qui consiste en un passage d'une culture à l'autre et dont les enjeux, les perspectives de développement et les applications sont nombreuses notamment dans un monde mondialisé avec ce qu'elle suppose comme volume de traductions spécialisées.

Constitué de trois grandes parties, la définition du domaine (son épistémologie et son histoire), les approches et théories de la traductologie et enfin ses applications, l'ouvrage brasse tous les aspects relatifs à l'étude de la traductologie permettant au lecteur de se former une idée générale de ce qu'est la traductologie dans ses différents volets. Chacun des dix chapitres qui constituent l'ouvrage est consacré à un aspect particulier : Délimitation du champ, Aperçu historique de la traduction, Approches et modèles de la traduction, Théories de la traduction, Questions et problématiques de la traduction, Traduction et interprétation, Pédagogie et didactique de la traduction, Champs d'application de la traductologie, et Traduction automatique.

Pratiquée depuis l'aube des temps, la traduction est une activité qui ne cesse d'évoluer et de se répandre au fil des évolutions politiques, économiques et culturelles des sociétés et, plus récemment, des progrès technologiques. Cependant, l'étude de la traduction comme activité, comme processus et comme résultat est beaucoup plus récente et encore en gestation. 
En retraçant l'histoire de la traduction, Guidère revient sur les raisons pour lesquelles la recherche en traductologie demeure timide. D'une part, le schisme entre praticiens et théoriciens du domaine a longtemps été tel que traduction et réflexion sur la traduction ne sont pas allées de pair. D'autre part, la traductologie a longtemps été rattachée à d'autres disciplines telles que la critique littéraire, la linguistique, l'informatique, les sciences cognitives et ce n'est que très récemment qu'elle s'est constituée en domaine indépendant.

La distinction que fait Guidère entre les approches et les théories de la traductologie développées depuis la seconde moitié du xxe siècle est un exemple parlant de cette évolution : les premières approches de la traduction, qui ont emprunté leurs outils à d'autres disciplines, comme les approches linguistiques, communicationnelles ou cognitives, ont laissé la place à des théories " strictement " traductologiques dont les plus connues sont la théorie du sens de l'École supérieure de traducteurs et d'interprètes (ESIT) de Paris, basée sur l'expérience de ses tenants en interprétation de conférences, et la théorie du Skopos qui conçoit la traduction comme un processus régi par son objectif (skopos en grec). Guidère le met bien en évidence tout au long de l'ouvrage, la traductologie a ses propres problèmes, enjeux et techniques même si elle emprunte parfois des outils conceptuels à d'autres domaines ; elle est ainsi un domaine fondamentalement interdisciplinaire.

La dernière partie, conséquente, de l'ouvrage est consacrée aux applications diverses et variées de la traductologie, qui sont une preuve vivante de l'étendue et du dynamisme de l'activité de la traduction : terminologie multilingue, lexicographie bilingue, veille multilingue, etc.

Sur le plan de la forme, la structure de l'ouvrage est particulièrement intéressante du point de vue didactique : trois rubriques synthétiques viennent clôturer chaque chapitre : "Faites le point " permet de retenir les idées centrales traitées dans le chapitre en récapitulant les points essentiels et en les mettant en perspective dans l'éventail large de la traductologie. "Pour aller plus loin " propose une bibliographie thématique sélective qui guide le lecteur vers des lectures plus approfondies. Enfin, la rubrique "Testez vos connaissances " contient une série de questions permettant d'évaluer ses acquis, une rubrique didactique particulièrement utile aux enseignants et aux étudiants de traduction.

Enfin, dans le souci de jeter les ponts entre les volets professionnel et académique de la traduction, l'auteur se pose en bon pédagogue en offrant un ouvrage de traductologie accessible aussi bien aux chercheurs qu'aux traducteurs professionnels et aux étudiants en traduction ou en sciences du langage. Cet ouvrage vient ainsi combler une lacune majeure pour les lecteurs francophones qui souhaitent s'initier au domaine riche, prometteur et méconnu qu'est la traductologie.

Introduction à la traductologie, Mathieu GUIDÈRE, Éditions De Boeck, Collection Traducto, Bruxelles 2008, 176 pages. 